\title{
Nutrition as Prevention and Treatment of Osteoporosis
}

\author{
M. STRÁNSKÝ ${ }^{1}$, L. RYŠAVÁ ${ }^{2}$ \\ ${ }^{1}$ Faculty of Public Health and Social Studies, South-Bohemia University, České Budějovice, ${ }^{2}$ State \\ Institute for Health, Prague, Czech Republic
}

Received June 18, 2009

Accepted July 14, 2009

\begin{abstract}
Summary
Osteoporosis is a systemic disease of the skeleton, characterized by reduction of bone mass and concurrent deterioration of bone structure. Consequently, bones are more fragile, and there is increased risk of fractures. The potential for acquisition of maximum bone mass is influenced by a number of factors. Among those are heredity, sex, nutrition, endocrine factors, mechanical influences and some risk factors. The best documented nutrient for metabolism of bone is calcium. Major role in the pathogenesis of osteoporosis have some micro and macro nutrients, prebiotics, alcohol, alternative diets, starvation and anorexia. Meta analysis of 29 randomized trials showed that supplementation with calcium and vitamin D3 reduces risk of bone fractures by $24 \%$ and significantly reduces loss of bone mass. Osteoporosis has multi factor etiology. Osteoporosis is one of diseases which are influenced by nutrition and life style. It is preventable by means of adequate nutrition and sufficient physical activity.
\end{abstract}

\section{Key words}

Osteoporosis • Nutrition • Macronutrients • Micronutrients • Prevention

\section{Corresponding author}

M. Stránský, South-Bohemia University, České Budějovice, Faculty of Public Health and Social Studies, Czech Republic. Tel.: +420 387315 181. E-mail: m.stransky@bluewin.ch

\section{Introduction}

Osteoporosis is a systemic disease of the skeleton, characterized by reduction of bone mass and concurrent deterioration of bone structure. Consequently, bones become more fragile and there is increased risk of fractures (Zittermann 2007, Scholz-Ahrens and Schrezenmeier 2004, Weichselbaum and Rieder 2007, Grashoff 2002). Osteoporosis is not exclusive to older age. It is however one of diseases preventable by adequate nutrition and physical activity.

Bone is a metabolically active organ. Puberty, as well as infancy is a period of life with most intensive bone growth: $90 \%$ of bone mass will have formed by the end of adolescence. In the first 5-6 years of life, around $100 \mathrm{mg}$ of calcium is retained daily for bone formation, up to $400 \mathrm{mg}$ or even more during puberty. After adolescence, absorption of calcium declines, in young adults daily absorption of calcium amounts to maximally $150 \mathrm{mg}$. Percentage of calcium absorption from the intestine reaches $75 \%$ in infancy, it is between 20-30\% in adults and declines further with increasing age (Scholz-Ahrens and Schrezenmeier 2004, Grashoff 2002). On these grounds, childhood and adolescence are important for optimal formation of bone and for prevention of osteoporosis in older age. After the age of 30 , there is no further increase of bone mass and a gradual natural reduction ensues.

\section{Factors influencing peak bone mass}

A number of factors influence peak bone mass (Zittermann 2007, Scholz-Ahrens and Schrezenmeier 2004, Burckhardt 2001, Osteoporosis Foundation 2003, Weichselbaum and Rieder 2007, Grashoff 2002). These include heredity, sex, nutrition, endocrine factors (sex hormones, calcitriol), mechanical influences (physical 
activity, body mass) and some risk factors (smoking, excessive alcohol intake, etc.). Genetic factors influence mainly absorption of calcium from the intestine: gene responsible for the formation of vitamin D receptors has several variants, which have different effect on absorption of calcium. Genetic differences can however be compensated for by adequate supply of calcium in diet (Grashoff 2002).

Deposition of calcium into bones is also under the influence of sex hormones, testosterone in men, estrogens in women. Late puberty, childlessness, early menopause, secondary amenorrhoea and ovarectomy are risk factors for osteoporosis in women. Peak bone mass and metabolism of calcium in bone are influenced by physical activity (Scholz-Ahrens and Schrezenmeier 2004, National Osteoporosis Foundation 2003, Grashoff 2002). Mechanical forces have essential role. Not so much weight bearing (body mass), rather pulling forces acting on entheses. Physically fit post-menopausal women have $25 \%$ higher intestinal absorption of calcium and calcitriol blood levels than unfit ones (Scholz-Ahrens and Schrezenmeier 2004). Among appropriate activities are resistance exercise, regular hiking, dance, gymnastics, which concurrently improve co-ordination of movements and hence help to prevent falls and injuries.

Calcium content in bone positively correlates with bone mass. Subjects with bone $\mathrm{BMI}<23$ have lower bone density than those with BMI $>27$. Women who are overweight or obese, have better absorption of calcium and lesser post-climacteric bone mass loss, than those with normal body weight. Women with lower BMI have higher risk of bone fractures caused by lower bone density and lesser amount of protective subcutaneous fatty tissue (Burckhardt 2005). For protection of osteoporosis in older women it is acceptable to be a little overweight, as long as there are no risk factors for cardiovascular disease (hypertension, dyslipidaemia, type 2 diabetes, etc).

\section{Intake, metabolism and excretion of calcium}

For primary and secondary prevention of osteoporosis, an important role has sufficient intake of calcium, as well as its optimal utilization by the organism. A number of factors influence the absorption of calcium as well as its excretion in urine (Zittermann 2007, Scholz-Ahrens and Schrezenmeier 2004, Burckhardt 2005, Weichselbaum and Rieder 2007, Grashoff 2002, Ilich et al. 2002). Absorption depends on the chemical form of calcium, the presence of other substances in food, physiological factors, amount of calcium in diet and timing of its ingestion during the day (Grashoff 2002). One of substances, which influence absorption of calcium most significantly is vitamin D, which increases its intestinal absorption and thus can, within limits, compensate for its lesser intake. By supplementation with 20 microgrammes of vitamin D, together with $1,200 \mathrm{mg}$ of calcium, it is possible to reduce risk of fractures. High doses of vitamin D, while increasing absorption of calcium by $12-13 \%$, increase its renal excretion. High doses of vitamin D are therefore not recommended (Scholz-Ahrens and Schrezenmeier 2004, Grashoff 2002).

Lactose increases intestinal absorption of calcium (Grashoff 2002). It is not exactly known why, but it may be because it changes $\mathrm{pH}$ in the small intestine. Glucose and Fructose increase its absorption too. Sweetened drinks, which are often consumed instead of milk, are no substitute for this important source of calcium. Prebiotics lactulose and oligofructose increase its absorption by $1 / 6-1 / 3$. This effect is believed to be caused by facilitation of increase in biphido bacteria, which then lower $\mathrm{pH}$ in the intestine, thus improving solubility of calcium (Grashoff 2002). Fat and fatty acids bind in the intestine to calcium to form soaps, which cannot be absorbed. There is however no known connection between high fat intake and increased risk of osteoporosis. Unsaturated fatty acids of omega- 3 type are believed to have protective effect on bone mineralization: their serum content correlates positively with bone density in vertebrae and the rest of the skeleton too. There is also correlation between bone mineralization up to the age 22-24 years and serum concentration of DHA (docosahexapentoic acid). Increased intake of omega-6 PUFA however probably reduces mineralization of pelvic bones (Zittermann 2007).

Diet low in protein is a risk factor for osteoporosis. It reduces calcium absorption and accelerates demineralization of bones in older age, particularly of vertebrae and the neck of femur. Amino acids with SH-group, which come predominantly from animal protein, reduce urinary $\mathrm{pH}$, thus reducing reabsorption of calcium in renal tubules. This negative effect has however no significant bearing on the risk of osteoporosis. Likewise, no difference has been observed between the incidence of fractures in vegetarians and subjects eating a mixed diet. Phospholipids, which are the product of cleavage of caseine during digestion, increase 
the solubility of calcium in the intestine and thus can increase the absorption of calcium (Zittermann 2004, Scholz-Ahrens and Schrezenmeier 2004). High Phosphate ingestion reduces the absorption and availability of calcium (Scholz-Ahrens and Schrezenmeier 2004, Grashoff 2002, Burckhardt 2005). In normal western diet, however, daily intake of Phosphate has no significant bearing on bone density. Salt increases urinary loss of calcium by $4-5 \%$ for each $500 \mathrm{mg}$ of sodium ingested in food. The activity of osteoclasts is however not affected Post-menopausal women are considerably more sensitive to excess of salt than younger women and consequently have to aim for low salt and high calcium diet.

Among foodstuffs which reduce intestinal absorption of calcium are whole grain products, particularly all bran, which contains phytates. They appear to have no impact on bone density, probably they increase reabsorption of calcium by the kidney. Apart from substances with negative effect on bone, whole grain foods contain substances, which have a beneficial effect on bone. Among those are lignans and phytoestrogens, contained particularly in soy (ScholzAhrens and Schrezenmeier 2004, Grashoff 2002). Phytoestrogens, by means of competitive binding to estrogen receptors, may have in higher doses similar effect to ovarian estrogens (Grashoff 2002).

Vitamin $\mathrm{K}$ has an important role in the formation of osteocalcin and other proteins specific for bone formation (Zittermann 2007, Burckhardt 2005). In postmenopausal women vitamin $\mathrm{K}$ significantly increases reabsorption of calcium in kidneys and reduces the risk of fractures. Vitamin A reduces bone density and increases risk of fractures of the hip (Zittermann 2007, Burckhardt 2005). The evaluation of Women's Health Study of 30,000 post-menopausal women showed however that the risk in women supplemented with this vitamin was raised only marginally. Also, newer NHANES I follow up study shows that low as well as excessive intake of vitamin $\mathrm{A}$ is associated with increased risk of hip fractures. Some other vitamins have direct or indirect effect on mineralization of bone (vitamins B6, B12, C).

Starvation increases loss of bone mass. That is caused by lower calcium intake and increased excretion, particularly by kidneys. Starvation leads to metabolic acidosis, which reduces calcium reabsorption in renal tubules. Supplementation with $5 \mathrm{~g}$ of calcium lactate daily during starvation compensates for the calcium deficit and keeps its metabolism in balance.
In anorexia, loss of calcium increases three fold. Supplementation with $150 \mathrm{mg}$ of calcium daily is not sufficient to compensate. Lack of estrogens leads to amenorrhoea. Hormonal changes are however not the sole cause of bone mass reduction. A number of nutrients, which participate in the metabolism of bone tissue, e.g. proteins, calcium, vitamin D, group B vitamins, etc. are in short supply, too. Successful treatment of anorexia results in rapid normalization of bio-markers of bone metabolism. Nevertheless, it takes several years to reach bone mineralization comparable to that of healthy subjects.

No beneficial effect of vegetarian diet on bone density has ever been proven. That is probably because of relatively high content of $\mathrm{SH}$-amino acids in cereals, rice, oats, nuts and seeds. Other factors are the content of fibre, phytates and oxalates in vegetarian diet, which promotes resorption of calcium and its secretion in urine.

High alcohol intake has adverse effect on bone mass. It reduces bone density and increases risk of fractures. It also reduces differentiation of osteoblasts and their activity, educes absorption of calcium from the intestine and conversion of vitamin D into its active form. Subjects with high alcohol intake usually also have a low intake of calcium. To date it has not been possible to ascertain exactly what level of alcohol intake is associated with adverse effect on bone. In recent studies, beneficial effect of moderate alcohol intake on bone density in older women has been found; highest density was associated with weekly alcohol intake between 29$57 \mathrm{~g} \quad$ (Zittermann 2007, Scholz-Ahrens and Schrezenmeier 2004, Ilich et al. 2002).

Postulated adverse effect of tea and coffee was reviewed in light of recent studies. Daily consumption of coffee up to $300 \mathrm{mg}$ of caffeine daily (3-5 dl) has no effect on bone density, as long as there is sufficient intake of calcium. Adverse effect has only been found in subjects with higher coffee intake and concurrent calcium intake of less than $750 \mathrm{mg} /$ day (Scholz-Ahrens and Schrezenmeier 2004, Ilich et al. 2002).

\section{Calcium and bone tissue}

Of all nutrients necessary for metabolism of bone and bone density, calcium is the best researched and documented one (Zittermann 2007, Scholz-Ahrens and Schrezenmeier 2004, Ilich et al. 2002, Burckhardt 2005, Bernecker 2005, National Osteoporosis Foundation 2003, Weichselbaum and Rieder 2007, Grashoff 2002). Its 
effect has been proven in many prospective studies, interventional and clinical trials, which all agree, that optimal calcium intake increases bone density in children and adolescents, maintains bone density in adults and slows down its loss in post-menopausal women. Children and adolescents, who often have insufficient calcium intake, grow faster when supplemented with calcium. Recent studies demonstrated, contrary to earlier beliefs, that benefits of calcium supplementation on bone density continue for one or more years after it finished.

Parameters of bone resorption show lower readings as early as 4 hours after oral supplementation with calcium. This is mediated through reduction of parathormone secretion. In old age, secretion of parathormone increases, with consequent release of calcium from bone. Beneficial effect of calcium and vitamin D supplementation on reduced risk of hip fractures in older subjects has been well documented.

Lower calcium intake does not necessarily result in osteoporosis: in some, the absorption and utilization of calcium is increased and its loss in urine and stool reduced. If, however its daily intake drops below $800 \mathrm{mg}$ daily, losses will exceed intake. The efficacy of calcium absorption is better when smaller quantities are presented for digestion. It is important to split daily dose of calcium to several smaller doses, a larger dose should be given in the evening, when the activity of cells incorporating calcium into bone is highest. Studies from Japan demonstrated, that evening supplementation with 1,000 $\mathrm{mg}$ of calcium reduces nocturnal activity of osteoclasts and reduces loss of bone mass. Such effect has not been observed with supplements of $200 \mathrm{mg}$. Administration of $450 \mathrm{mg}$ of calcium at bed time reduces urinary excretion of bone resorption markers. To date, there has been no study to show that splitting daily requirement of calcium into several smaller doses has a beneficial effect on risk of fractures caused by osteoporosis. Evening supplementation with calcium in primary prevention of osteoporosis does not seem appropriate, but evening meal rich in calcium is to be recommended in both, primary and secondary prevention (Zittermann 2007, ScholzAhrens and Schrezenmeier 2004, Bernecker 2001).

Numerous other factors, apart from calcium and vitamin D play an important role in the pathogenesis of osteoporosis (Ilich et al. 2002). Zinc is important for synthesis of metaloenzymes for production of bone matrix, physical activity increases absorption of calcium in the intestine and increases bone density. Smoking is a risk factor (Zittermann 2007, Scholz-Ahrens and
Schrezenmeier 2004, Osteoporosis Foundation 2003, Ilich et al. 2002, Burckhardt 2005, National Osteoporosis Foundation 2003, Weichselbaum and Rieder 2007, Grashoff 2002).

\section{Interventional studies}

Numerous studies showed, that supplementation with calcium and vitamin $\mathrm{D}$ reduces the incidence of fractures: in one study 3,270 old women (average age 84 years) were supplemented with $1,200 \mathrm{mg}$ of calcium and 20 microgrammes of vitamin D3 for 18 months. Their risk of fractures of the neck of femur was reduced by $40 \%$ compared with controls. Similar results were published by Dawson-Hughes et al. (1997), who supplemented 246 subjects (average age 71 years) with $500 \mathrm{mg}$ of calcium and $17.5 \mathrm{mg}$ of vitamin D3 for 3 years. They found reduction in incidence of fractures in supplemented subjects by $50 \%$. Recently published meta-analysis of 29 randomised studies, which included a total of 63,897 women over the age of 50 years, showed reduction in the risk of fractures by $24 \%$ in women supplemented with $1,200 \mathrm{mg}$ calcium daily. A significant reduction in the loss of bone mass has been found as well.

The study by Dr. Tang et al. (2007) and colleagues, from the University of Western Sydney, used meta-analysis techniques to collate information from different studies of calcium supplementation. The data comprised 17 studies on bone fracture risk involving 53000 subjects, and 23 studies on bone density. Subjects were over 50 years and had been given additional calcium, often in conjunction with vitamin $\mathrm{D}$, for 3.5 years on average.

The results showed that people who had calcium intakes close to the recommended levels of 800-1000 mg per day were less likely to experience bone fracture. The effect was dose-dependent in that higher calcium intakes, of around $1200 \mathrm{mg}$ per day, reduced bone fracture risk to a greater extent than lower calcium intakes. Elderly people, those living in institutions, and those with a low body weight appeared to benefit the most from additional calcium. Adding extra vitamin $\mathrm{D}$ to the calcium-rich diet increased the bone protection, but not to a large extent.

\section{Prevention of osteoporosis - practical recom- mendations}

- consume daily at least 3 portions of milk and dairy produce with reduced fat content 
- choose vegetables with high calcium content (broccoli, cabbage)

- drink mineral water with high calcium content

- moderate your alcohol intake

- eat fish at least once a week

- limit consumption of foods and drinks high in phosphates

- use spices in place of salt to enhance flavor (chives, parsley)

- eat vegetables and fruit five times daily

- limit consumption of foods high in oxalates

- ensure sufficient intake of vitamin D (fish, livers, milk), vitamin K (leafy vegetables, livers, fish) and vitamin C

- engage in regular physical aktivity

- do not smoke

- limit salt intake

\section{Conclusions}

Osteoporosis is a multifactorial disease, the risk of which can be reduced by adequate nutrition and appropriate lifestyle. Prevention should start in childhood, as that is the time, when bone formation is very intensive and achievement of optimal peak bone mass is a necessary requirement for optimal bone density in older age. Majority of the above recommendations apply to all age groups, though they have different reasons at different ages. Optimal intake of calcium, vitamins $\mathrm{D}$ and $\mathrm{K}$ are important factors in primary as well as in secondary prevention of osteoporosis. Kidneys and the intestine have a limited capability to adjust the metabolism of calcium in response to actual requirements of the organism. Nutritional factors, which influence its absorption and excretion, need not necessarily have to influence its metabolism. When certain substances in food reduce intestinal absorption or increase excretion by the kidney, the organism can utilize compensatory mechanisms, to prevent adverse effect on bone.

\section{Conflict of Interest}

There is no conflict of interest.

\section{References}

BERNECKER P: Kalzium und Vitamin D in der Therapie der Osteoporose. J Ernährungsmed 3: 41-42, 2001.

BURCKHARDT P: Die Bedeutung der Ernährung in Prävention und Therapie der Osteoporose. SAR 4: 48-52, 2005.

DAWSON-HUGHES B, HARRIS SS, KRALL EA, DALLAL GE: Effect of calcium and vitamin D supplementation on bone density in men and women 65 years of age and older. $N$ Engl J Med 337: 670-676, 1997.

GRASHOFF K: Osteoporose - eine pädiatrische Erkrankung. Ernährungs-Umschau 49: 367-370, 2002.

ILICH JZ, BROWNBILL RA, TAMBORINI L, CRNCEVIC-ORLIC Z: To drink or not to drink; how are alcohol, caffeine and past smoking related to bone mineral density in elderly women? J Am Coll Nutr 21: 536-544, 2002.

NATIONAL OSTEOPOROSIS FOUNDATION. Boning up on osteoporosis: a guide to prevention and treatment. Washington, D.C. National Osteoporosis Foundation, 2003.

SCHOLZ-AHRENS KE, SCHREZENMEIR J: Ernährung und Osteoporoseprävention. Ernährungs-Umschau 51: 2226, 2004.

TANG BM, ESLICK GD, NOWSON C, SMITH C, BENSOUSSAN A: Use of calcium or calcium in combination with vitamin $\mathrm{D}$ supplementation to prevent fractures and bone loss in people aged 50 years and older: a metaanalysis. Lancet 370: 657-666, 2007.

WEICHSELBAUM E, RIEDER A: Österreichischer Osteoporosebericht. Verein Altern mit Zukunft. Wien, 2007.

ZITTERMANN A: Osteoporose. Ernährungs-Umschau 54: B 33-38, 2007. 